\title{
Research on Splicing and Three-dimensional Measurement of Hydraulic Turbine Blades Surface
}

\author{
Yang Ling and Yuan Jiahe \\ The Higher Educational Key Laboratory for Measuring \& Control Technology \\ and Instrumentations of Heilongjiang Province, Harbin University of Science and \\ Technology, Harbin, China, \\ lindaleg9@126.com
}

\begin{abstract}
The splicing and three-dimensional measurement of the hydraulic turbine blade is achieved in this paper. The large size of hydraulic turbine blades makes it impossible to take a complete picture of the entire blade. Multiple images of various segments must be obtained and then spliced together at later stages. Landmarks are installed in public area of the adjacent segments of hydraulic turbine blades, which are used when splicing segmental images together using least squares method, to obtain three-dimensional surface information ofthe entire blade. Based on the principles of the structure light measurement, a three-dimensional measurement system is designed, its parameters are also defined, and mathematical models of the three-dimensional measurement are established. The hydraulic turbine blade is simulated by using software package " $3 d s$ max" to build a hydraulic turbine blade model. Simulation of splicing and threedimensional measurement of the hydraulic turbine blade shape is achieved. The reconstruction of the three-dimensional Hydraulic turbine blade is then obtained based on the measured data.
\end{abstract}

Keywords: splicing, three-dimensional measurement, hydraulic turbine blade, landmark

\section{Introduction}

Hydraulic turbine blades are an absolute essential part of large hydropower equipments. Accurate measurement of the blade has crucial impact on the overall operational efficiency and services. As the demands for the output power and efficiency continuously increase, the profile dimension of the hydraulic turbine blades are increasing, the demands for specifications and precision of hydraulic turbine blades are also increasingly higher. We often encounter the situation where it is not possible to acquire the entire data set of three-dimensional measurement of those blades [1].

In this paper, we used a segmentation photography method to obtain segmented images of a entire blade. The three-dimensional parameters were then extracted from those images and spliced together to reconstruct three-dimensional surface information of the blade in its entirety.

\section{The Principle of Three-dimensional Measurement of Hydraulic Turbine Blades}

In this paper, we used a common measuring method know as Structure Light Threedimensional Measuring method [2, 3]. This is based a well-known mathematical theorem: For a given triangle, if two angles and one side are known, we can then solve the triangle (calculate the other two sides and the remaining angle). 
In the following diagram (Figure 1), the cylinder represents a blade. A beam of encoded light is projected from the projector (DLP) located at $O_{1}$ to a point $Q(x, y, z)$ on the blade. The image is captured by a camera located at $O_{2}$. We know the distance between the projector and the camera $\left(O_{1} O_{2}\right.$, which will be referred to as the "base line"). We also know the associated various angles between the projected light and the baseline, as well as between the line of sight of the camera and the baseline. We then now calculate all other sides we need.

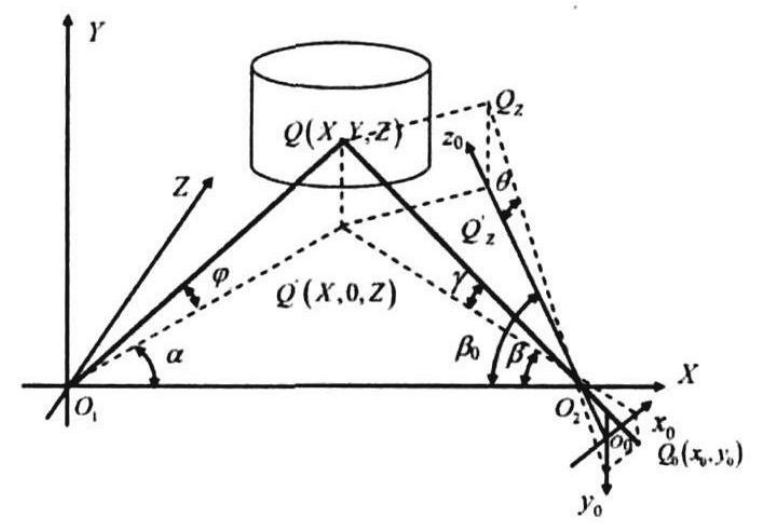

Figure 1. The Theory of Measuring on Color-encoded

$O_{1}$ is the center point of the lens of the DLP; $O_{2}$ is the center point of the lens of the camera. $O_{1} O_{2}=B$; Point $Q$ is the spot up on which the encoded light projected to. The point $Q$ is on the coordinate system $X Y Z O_{1}$, The point $Q_{0}$ is the image of $Q$ as captured by the camera, and it is on the image surface $X_{0} O_{0} Y_{0}$ of the coordinate system $X_{0} Y_{0} Z_{0} O_{0}$. $X_{0} O_{0} Z_{0}$ is on the same surface as $X O_{1} Z . Q^{\prime}, Q_{\mathrm{z}}$ and $Q_{\mathrm{z}}^{\prime}$ are images of spot $Q$ on the surface $X O_{1} Z, Y_{0} O_{0} Z_{0}$ and the axis $O_{0} Z_{0}$ respectively. Based on the triangulation principle, the $3 \mathrm{D}$ coordinates $(x, y, z)$ of the point $Q$ on the object surface can be calculated using formulas (1), (2) and (3). In formulas (1), (2) and (3), $f$ represents the focus of the camera's lens, $\beta_{0}$ is the angle between the optic axis $O_{0} Z_{0}$ and the base line $O_{1} O_{2} . f, B$, and $\beta_{0}$ are constants. $x_{0}, y_{0}$ are determined by the pixels of CCD in the camera. Angle $\alpha$ is the projection angle.

$$
\begin{gathered}
Z=\frac{B}{\cot \alpha+\frac{x_{0}+f \cot \beta_{0}}{f-x_{0} \cot \beta_{0}}} \\
Y=\frac{Z \cdot y_{0}}{f \sin \beta_{0}-x_{0} \cos \beta_{0}} \\
X=Z \cot \alpha
\end{gathered}
$$

\section{Landmarks Three-dimensional Splicing Techniques}

While measuring the surface of hydraulic turbine blades, due to their large size, it often becomes necessary to measure the blades in segments. The segmented measurement data can then be spliced together through image processing techniques. That is the idea of "three-dimensional shape splicing technology".

In this paper, we discussed a technique of projecting landmarks with identifiable characteristics on to the blade surface. Those landmarks can then be easily identified and matched later while stitching the segmented data together using coordinate transformation matrix. This is the so called Land Mark Based Splicing Method [4-5]. 


\subsection{Image Splicing Theory}

First of all, let us assume that the measurement of the blade must be done in two stages in order to establish the model. This would require that the blade must be measured in two fields of view. The captured images in the two fields of view must have an overlapping portion [6]. There are two projectors in this measuring system, the first projector projects the color coded stripe patterns to the blade, the second projector projects a number of landmarks to the blade. After the camera obtains images in the first field of view, the first projector and the camera must be moved synchronously to the second field of view. Coordinates of the blade surface can then be measured using these two images of the same blade with the color code stripe pattern obtained by the camera in the two fields of view. By identifying and matching the land barks in both fields of view and images registration, the three-dimensional coordinates of the landmarks can be obtained. We can then solve for the coordinate transformation matrix between them based on the variation model of the blade, and use it stitch the images together.

\subsection{Three-dimensional Coordinate Transformation}

Three-dimensional coordinate transformation is completed through a generalization of a two-dimensional coordinate transformation. A $3 \times 3$ matrix can be used to express a two-dimensional coordinate transformation. Let $P$ be a point in a two-dimensional space with coordinates $(x, y)$. Let $h$ be a non-zero constant. Then the homogeneous coordinates of the point $P$ can be expressed as $\left(x^{\prime}, y^{\prime}, h\right)$, where $x^{\prime}=h x, y^{\prime}=h y$. For simplicity, let us assume that $h$ is 1 , then the homogeneous coordinates of the point is $\left(x^{\prime}, y^{\prime}, 1\right)$. Its two-dimensional linear transformation form is usually written as in formula (4).

$$
\left[\begin{array}{lll}
x^{\prime} & y^{\prime} & 1
\end{array}\right]=\left[\begin{array}{lll}
x & y & 1
\end{array}\right]\left[\begin{array}{lll}
a_{1} & a_{2} & a_{3} \\
b_{1} & b_{2} & b_{3} \\
c_{1} & c_{2} & c_{3}
\end{array}\right]
$$

The general form of this transformation is the formula (5), the original coordinates of the point is expressed as $Q$, The transformed coordinates is expressed as $Q$. The transformation matrix is expressed as $T$. The matrix $T$ varies according to transformations

$$
Q^{\prime}=Q T
$$

Similarly, the three-dimensional coordinate transformation of a point in a threedimensional space can be expressed as a $4 \times 4$ homogeneous transformation matrix .Let $(x$, $y, z)$ be the coordinates of a point in a three-dimensional space, and let $(x y z 1)$ be its homogeneous coordinates, then the three-dimensional linear transformation can be expressed as formula (6), where Formula (7) is its corresponding three-dimensional coordinate transformation matrix.

$$
\left(x^{\prime}, y, z^{\prime}, 1\right)=(x, y, z, 1)\left[\begin{array}{llll}
S_{11} & S_{12} & S_{13} & S_{14} \\
S_{21} & S_{22} & S_{23} & S_{24} \\
S_{31} & S_{32} & S_{33} & S_{34} \\
S_{41} & S_{42} & S_{43} & S_{44}
\end{array}\right]
$$




$$
S_{3 D}=\left[\begin{array}{llll}
S_{11} & S_{12} & S_{13} & S_{14} \\
S_{21} & S_{22} & S_{23} & S_{24} \\
S_{31} & S_{32} & S_{33} & S_{34} \\
S_{41} & S_{42} & S_{43} & S_{44}
\end{array}\right]
$$

The three-dimensional coordinate transformation matrix $S_{3 D}$ can be divided into four sub-matrices according to their respective functionalities:

$$
\left[\begin{array}{lll}
s_{11} & s_{12} & s_{13} \\
s_{21} & s_{22} & s_{23} \\
s_{31} & s_{32} & s_{33}
\end{array}\right] 、\left[\begin{array}{lll}
s_{14} & s_{24} & s_{34}
\end{array}\right] 、\left[\begin{array}{lll}
s_{41} & s_{42} & s_{43}
\end{array}\right] \text { and }\left[s_{44}\right] .
$$

The sub-matrix $\left[\begin{array}{lll}s_{11} & s_{12} & s_{13} \\ s_{21} & s_{22} & s_{23} \\ s_{31} & s_{32} & s_{33}\end{array}\right]$ represents the linear transformation, including local scaling, shear, rotation and reflection.

The sub-matrix $\left[\begin{array}{lll}s_{14} & s_{24} & s_{34}\end{array}\right]$ represents the projective transformation;

The sub-matrix $\left[\begin{array}{lll}s_{41} & s_{42} & s_{43}\end{array}\right]$ represents the translation along the $x, y, z$ direction respectively;

The sub-matrix $\left[s_{44}\right]$ represents the overall scaling factor.

When measuring Segmentation in this paper, only pan the blade model in the two fields of the view, So just considering the sub-matrix $\left[\begin{array}{lll}S_{41} & s_{42} & S_{43}\end{array}\right]$.

Assuming that a pixel of the blade model image data in a visual field coordinate $A=\left(x_{1}\right.$ $\left.y_{1} z_{1} 1\right)$, its data coordinate in field 2 is $B=\left(x_{2} y_{2} z_{2} 1\right)$, then the relationship between these two coordinates can be expressed by the formula (8) the same point coordinates transformation in different field can be made through the formula.

$$
A S_{3 D}=B
$$

\subsection{Stitching Algorithm Steps}

The data splicing procedure is divided to the following steps based on least-squares method.

The first step, the pixel coordinates of the landmark are extracted by binarization processing the blade images which are in two fields of the view. The two corresponding landmarks are registration using landmark recognition technology. If the right number of landmark point is more than three pairs after registration, then to splice. The matrix of their coordinates is written $P$ and $Q$.

The second step, according to the formula $S_{3 D}=P^{+} Q$ to calculate the coordinate transformation matrix $S_{3 D}, P^{+}$represents $P$ generalized inverse, $P^{+}=P^{T}\left(P P^{T}\right)^{-1}$.

The third step, the landmark pixel coordinates in different fields of view are written into matrix $P$ and $Q$, and into the formula to achieve splicing.

According to the splicing theory, the more right landmarks are registration, the less error and the finer stitching.

\subsection{Setting Landmarks}

Three-dimensional color-coded structured light measurement method is used in this paper. Setting the first view field, simulation projector is replaced by the target spotlight, 
being shown as Figure 2.The spotlight is placed in the origin of the world coordinate system $O_{1}$, the angle between the optical axis of the projector and the x-axis is $60^{\circ}$, and the opening angle of the projector is $60^{\circ}$. Simulation camera placed at position away $x$ axis $900 \mathrm{~mm}$ from the projector, the angle between its optical axis and the $x$-axis is $60^{\circ}$, and the opening angle is $60^{\circ}$.Due to the larger blade model, according to the actual situation, the blade model is placed in the center of the $(800,900,24.57)$ which get the optimal effect [7].

The projector 2 is placed in the $x$-axis, coordinates $(800,0,0)$ for projecting landmarks in simulation system, as shown in Figure 3.

Designing the second view Field, the relative position of the projector 2 and blade model should remain unchanged. The blade model and projector 2 are panned560mmto the left, $x$-axis coordinates of both the blade model and the projector 2changed to 240, Figure 4 shows the schematic view.

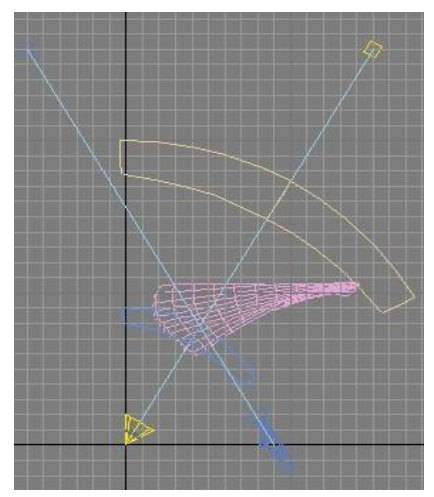

Figure 2. The Settings in the First Field of View

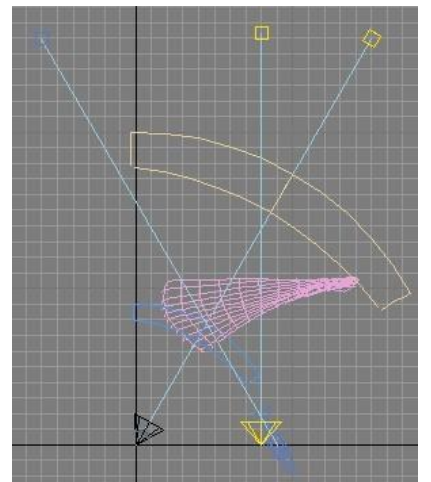

Figure 3. Projector 2 in the Simulation System

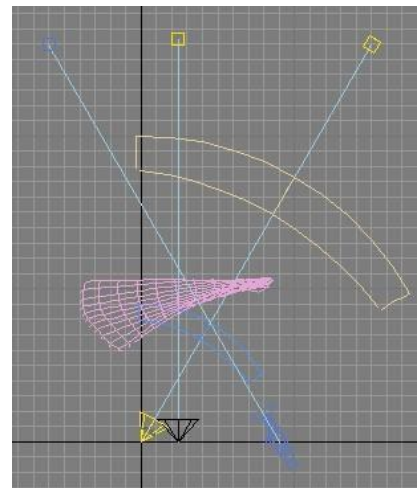

Figure 4. The Settings in Field of View 2 
After the binarization processing on the color coding pattern of two view fields, decoding the color-coded pattern, three dimensional coordinate value of the point on the blade surface can be measured by decoding, and the three-dimensional shape can be reconstructed.

\section{Detection Technology of the Circular Landmark}

Hough transformation is one of the basic geometry methods which identified from the image processing [8-9], it is edge connection technology which based on the image global segmentation result. Its basic theory is that utilize the duality between point and line. Making the lines of the image space into a rallying point of the parameter space, which changing the original image that is given curve detection problem into finding the peak of the problem parameter space. As convert the overall characteristics of the detection to detect local properties. Such as lines, ovals, circles and arcs, etc. It has strong antijamming capability. It can be detected in any shape curve even if there are many fracture traces. It is widely used in many fields of biomedical, automation, robot vision, space technology, military defense, and office automation. In recent years, Hough transform achieved satisfactory results in terms of circle detection [10], the circle detection algorithm receives a higher practical value which based on Hough transformation.

An improved Hough transform [11] circle detection algorithm is adopted in this paper. In figure 5, $V$ is the gradient in the vertical direction; $H$ is a gradient in the horizontal direction.

Firstly, $a, b, c, d$ are the four points on the circle, $a, b$ and $c, d$ are the $y$-axis symmetry respectively. $a, d$ and $b, c$ are $x$-axis symmetry respectively, the gradient magnitude and same direction on the vertical direction of the $y$-axis of symmetry, the same gradient magnitude in the horizontal direction but opposite direction, the gradient magnitude and the same direction on the vertical direction of the $x$-axis of symmetry, the same gradient of the vertical direction but opposite direction.

Secondly, connecting $a, b$ and $c, d$ as well as $a, d$ and $b, c$ respectively, finding the perpendicular bisector, the intersection of the several perpendicular bisector is the center of the circle. It has the same direction with the gradient direction of each point of symmetry in the horizontal direction, opposite direction of the gradient in the vertical direction.

In schematic figure6, identify the point $A$ in the circle according to the size of the gradient in the vertical direction on the $y$-axis of symmetry of the point which has the same direction, the same size of the gradient in the horizontal direction and the opposite direction of the law, there is a point $B$ in the horizontal direction on the right. Connecting $A$ and $B$, calculating $A B$ midpoint $C$, midpoint $C$ is on the vertical line $A B$, chord $A B$ in the middle vertical must exist point $O$, that is the center of the circle. Calculating $D\left(x_{d}, y_{d}\right)$ along the negative direction of the $y$-axis at intervals of 1 . Connecting $A, D$ or $B$, $D$,its distance is calculated, accumulating $\left(x_{d}, y_{d}, r\right)$ in the parameter space. Accumulating the next point $E$ with unit 1 as interval, stop to find when the value exceeds the $y$ or $r$ which is set in advance, and start looking for the next point $A$ and the point $B$. 


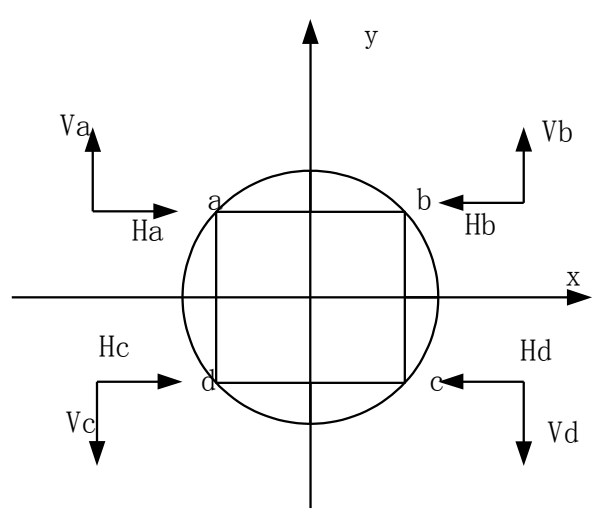

Figure 5. Schematic Diagram of the Center Position

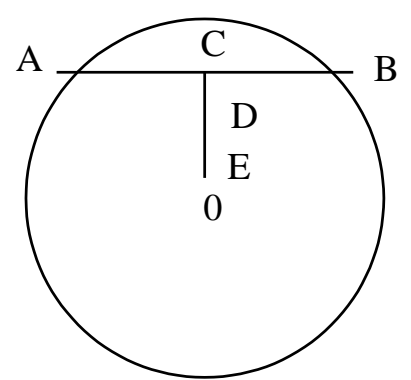

Figure 6. Circle Detection Method Schematic

Figure 7 shows the blade model images which are projected the landmarks by projector 2 in two fields of the view respectively.

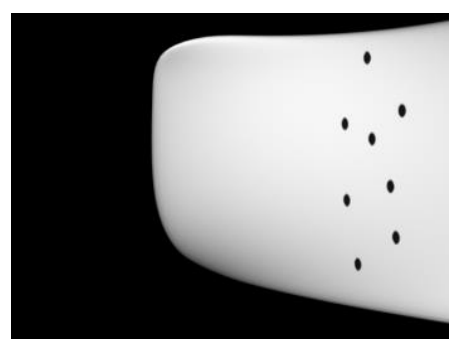

a) landmarks image in the first field of view

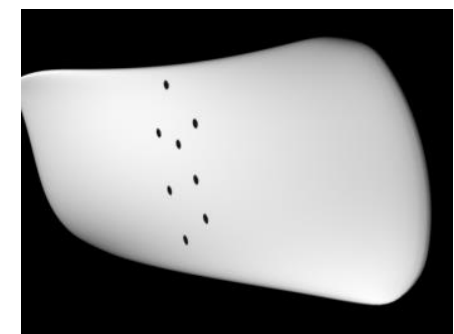

b) landmarks image in the second field of view

Figure 7. Shooting Images in Two Fields of View

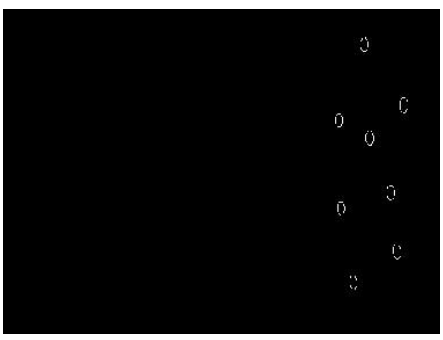

a) Edge

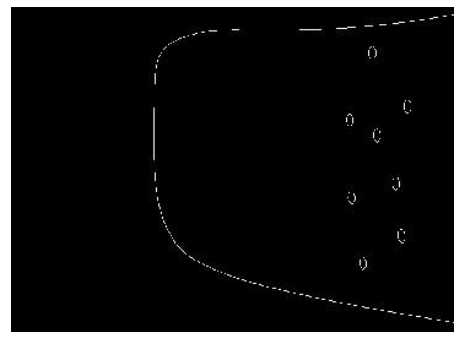

b) detecting a pie chart

Figure 8. Image Circle Detection in Field 1 of View

Processing the object image which contains the landmarks circle in the first field of view, after using the hough transform circle detection, the tested results are shown in figure 8 and the detected center coordinate is shown in Table 1.

Table 1. Detected Center Coordinates

\begin{tabular}{l|l|l|l|l|l|l|l|l}
\hline $\mathrm{X}$ & 489 & 491 & 506 & 520 & 527 & 554 & 562 & 572 \\
\hline $\mathrm{Y}$ & 164 & 276 & 370 & 66 & 185 & 255 & 331 & 144 \\
\hline Radius & 6 & 6 & 6 & 6 & 6 & 6 & 6 & 6 \\
\hline
\end{tabular}

Similarly, the same processing is done with captured landmarks circle image in the second field of view. The tested results are shown in Figure 9. 


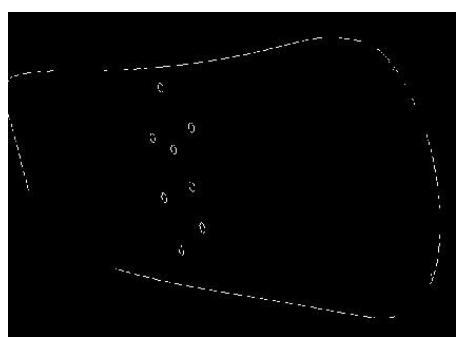

a) Edge

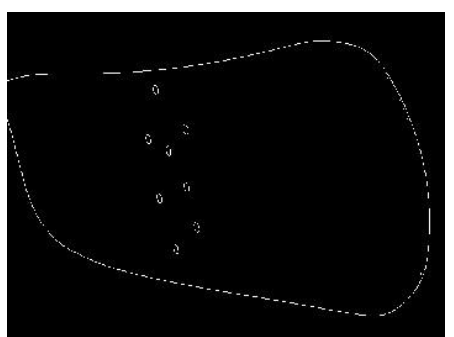

b) detected circular

Figure 9. Circle Detection in the Second Field of View

The detected center coordinates are shown in Table 2.

Table 2. Detected Center Coordinates

\begin{tabular}{l|l|l|l|l|l|l|l|l}
\hline $\mathrm{X}$ & 216 & 259 & 205 & 234 & 221 & 260 & 245 & 274 \\
\hline $\mathrm{Y}$ & 112 & 169 & 183 & 200 & 268 & 252 & 341 & 310 \\
\hline Radius & 6 & 6 & 6 & 6 & 6 & 6 & 6 & 6 \\
\hline
\end{tabular}

Accordance with the registration principle of the landmarks circle, after the landmarks circle registration, the coordinate transformation matrix is calculated under both fields of view according to the least squares method.

$$
S_{3 D}=\left[\begin{array}{cccc}
0.5389 & 0.2230 & 1.7057 & 0.0007 \\
0.0798 & 0.4508 & -0.6264 & -0.0002 \\
0.1700 & -0.2361 & -0.4752 & -0.0002 \\
-430.0805 & 190.4295 & 928.9358 & 1.0000
\end{array}\right]
$$

Reconstruction of splicing results under two fields of view are shown in Figure 10.
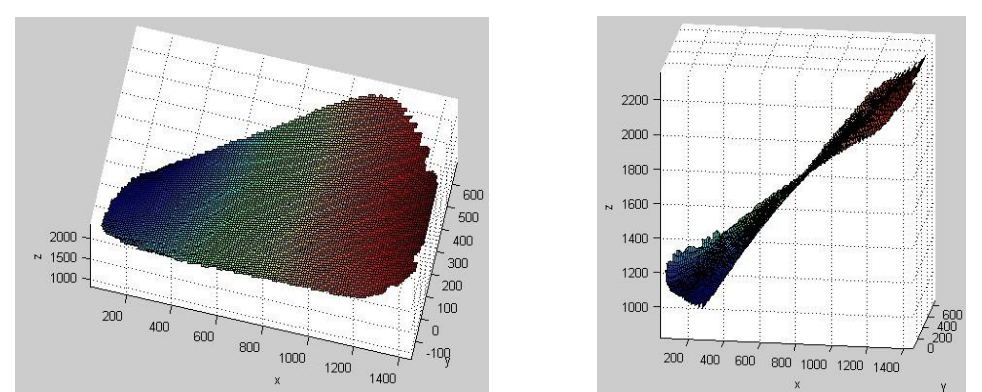

Figure 10. Splicing Result of Image under Both Fields of View

\section{Conclusion}

Experimental results show that the sectional measurement splicing method which used in this paper is simple and feasible, easy to operate. However, the error still exists when using the Hough circle detection technology. In order to get greater accuracy in future measurement, the Hough center of the detection accuracy needs be further improved.

\section{References}

[1] Q. Shu and D. Tang, "Path Trajectory Planning of Manipulator for Repairing Hydraulic Turbine [J]", Journal of Harbin University of Science and Technology, vol. 1, (2014), pp. 16-19. 
[2] H. Yuan, "Combination of color stripe encoding three-dimensional measurement techniques [D]", vol. 7, (2006).

[3] Y. Rao, Y. Wang, S. Yu, H. Wu, X. Yu and D. Chen, "Journal of Harbin University of Science and Technology, vol. 3, (2011), pp. 78-81.

[4] Y. Liang, W. Deng, X. Lou and N. Lu, "Automatic registration m method of multi-view 3D data based on marked points [J]", Journal of Beijing Information Science and Technology University, vol. 3, (2010), pp. 31-33.

[5] G. Wang, Y.-x. Zhong, C.-l. Yuan, Y.-b. Ma and R.-j. Li, "Study on 3D data connection of large part in 3 D noncontact measurement [J]", Machinery Design \& Manufacture, vol. 9, (2007), pp. 90-92.

[6] X.-j. Qu, Y. Wang, L.-x. Fan, S.-p. Li and Z.-r. Han, "Alignment of multi-view data of linear structure light scanning measurement [J]", Journal of Plasticity Engineering, vol. 10, (2012), pp. 124-129.

[7] Y. Ma, Y. Zhong, L. Zheng and C. Yuan, "Design and recognition of coded targets for 3-D registration [J]", Journal of Tsinghua University(Science and Technology) vol. 46, no. 2, (2006), pp. 169-175.

[8] B. Wang and X. Huang, "Hough Transform and Application in Geometrical Property Detection [J]", Computer and Modernization, vol. 4, (2008), pp. 20-22.

[9] X. Zhang and Pengwei, "Circular object detection based on Hough transform [J]", Sensors and Microsystems, Sensors and Microsystems, vol. 25, no. 4, (2006), pp. 62-64.

[10] C. Kimme, D. Balland and J. Sklansky, "Finding circles by an array of accumulators [J]", Commun., ACM, vol. 15, no. 1, (2007), pp. 11-15.

[11] L. Liu and Y. Wang, "A circle detection method based on Hough transform [J]", Microcomputer Information, vol. 5, (2009), pp. 274-276. 
International Journal of Multimedia and Ubiquitous Engineering Vol.10, No.4 (2015) 